\title{
Avalanches in fine, cohesive powders
}

\author{
Jose Manuel Valverde, Antonio Castellanos,* and Antonio Ramos \\ Departmento de Electronica y Electromagnetismo, Universidad de Sevilla, Avenido Reina Mercedes s/n, 41012 Sevilla, Spain \\ P. Keith Watson \\ Xerox Corporation, 800 Philips Road, 0114-22D, Webster, New York 14580
}

(Received 27 January 2000; revised manuscript received 24 May 2000)

\begin{abstract}
We have investigated the onset of avalanches in fine, cohesive granular materials. In our experiments shear stress is generated by tilting an initialized bed of powder and increasing the angle of tilt until the powder avalanches. We find that the angle $\alpha$ of the avalanche decreases with increasing bed width. The avalanche depth increases with the bed width and, in all cases, is of the order of several millimeters, which is much greater than the particle size. We carry out a macroscopic analysis of the avalanche process based on Coulomb's method of wedges. This analysis shows the fundamental role played by powder cohesion and boundary conditions on avalanches in fine cohesive powders. This behavior contrasts with the behavior of noncohesive grains, such as dry sand, where avalanches consist of superficial layers of about ten grains. The reason behind this is that for our experimental powders (particle diameter $\sim 10 \mu \mathrm{m}$ ) the van der Waals interparticle adhesive force exceeds several orders of magnitude particle weight. Adhesive forces oppose gravity, and as a result fine cohesive powders settle in very open structures as compared to noncohesive granular materials. Because of the dominance of adhesive forces over particle weight, our materials behave more like wet sand.
\end{abstract}

PACS number(s): 45.70.Ht, 83.70.Fn, 45.70.Mg, 81.05.Rm

\section{INTRODUCTION}

Avalanches in cohesiveless granular media have often been investigated [1-3]. A common characteristic of these studies is that granular motion occurs in a relatively thin boundary layer (around ten grains) at the surface [4], independently of the size of the sample. The reason for this behavior is the lack of cohesion between grains. This allows one to analyze the stability of slopes, based on frictional forces between individual grains. Several studies are dedicated to understanding the effect of cohesion induced by moisture in the angle of repose of the material [5-7]. In these studies the particle size is larger than $1 \mathrm{~mm}$, and the cohesive force between grains is lower than or at most comparable to the weight of the particles. The importance of weight still allows one to analyze the stability of slopes by means of the stability of two particles connected by a liquid bridge independently of boundary conditions [6]. However, when cohesive forces are much greater than the weight it is no longer possible to describe the avalanches by analyzing the behavior of individual grains. A familiar example of moisture induced cohesion is wet sand. Typical diameters of sand particles range from 100 to $500 \mu \mathrm{m}$, and particle volume can be reduced by three orders of magnitude with respect to a particle volume of 1-mm-sized particles. Interparticle capillary forces in wet sand can exceed the particle weight in 2-3 orders of magnitude [8]. As a consequence, cohesive effects are dominant in the bulk behavior. Wet sand has internal slip surfaces, with macroscopic blocks of wet sand detaching from the bulk, and the sample size determines the slope stability (all of us know by experience that the higher the sand castle the greater the chances of failure). Our mate-

\footnotetext{
*Corresponding author. Email address: castella@cica.es
}

rials are made of dry fine particles of diameter of the order of $10 \mu \mathrm{m}$. Dispersion or van der Waals forces, electrostatic forces, and capillary forces are regarded as the main forces in particle adhesion. We have minimized capillary and electrostatic forces, working with uncharged particles in an ambient of dry nitrogen gas. Consequently, van der Waals forces are dominant between our dry fine particles. These forces arise as a result of the interaction of molecules through fluctuating dipole-dipole fields. Summing up all the interactions between two spherical and rigid particles with diameters $d_{1}$ and $d_{2}$ leads to an attractive force [9]

$$
F_{v d W}=\frac{A}{12 z_{0}^{2}}\left(\frac{d_{1} d_{2}}{d_{1}+d_{2}}\right)
$$

where $A$ is the Hamaker constant, and $z_{0}$ is the distance of closest approach between two molecules which is between $3.0 \AA[10]$ and $4.0 \AA$ [11]. Most powder particles have a rather rough surface, with many asperities of the radii of curvature often not larger than $0.1 \mu \mathrm{m}$. Thus the effective contact area is given by the asperities at contact, and to calculate the interparticle adhesion the diameter of the asperities $d_{a}$ should be inserted into Eq. (1). For xerographic toners typical values are $A \approx 0.5 \times 10^{-19} \mathrm{~J}$ and $d_{a} \approx 0.2 \mu \mathrm{m}$, resulting in a value for the interparticle cohesive force $F_{v d W}$ $\sim 10^{-9} \mathrm{~N}$. For a typical density value $\rho_{p} \sim 1000 \mathrm{~kg} / \mathrm{m}^{3}$, the van der Waals force overcomes the particle weight for particle diameters $d_{p}$ of less than about $100 \mu \mathrm{m}$. For xerographic toners $\left(d_{p} \simeq 10 \mu \mathrm{m}\right)$ the van der Waals force can be up to four orders of magnitude higher than the particle weight [12-14]. Therefore, for these fine powders we expect a behavior more similar to wet sand than to dry sand, and we must consider boundary conditions and macroscopic stresses 


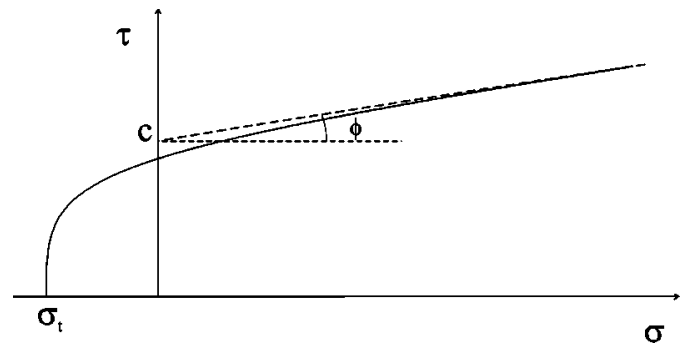

FIG. 1. Typical yield locus for a cohesive powder.

on their yield. For this reason we choose a continuum approach to analyze the problem.

In the continuum approach the onset of flow is characterized by an empirical limiting stress function, the yield locus, such that stresses lower than this limit cause negligible deformation, whereas at this limit there will be a combination of shear and compression stresses that will trigger the avalanche. At high stresses the yield locus can be approximated by a linear function known as Coulomb's law [15] shown in Fig. 1,

$$
\tau=\sigma \tan \phi+c,
$$

where $\tau$ and $\sigma$ are the tangential and normal stresses over the slip plane (i.e., the plane over which the yield condition is satisfied), and $c$ and $\phi$ are the cohesion and the angle of internal friction. For many coarse materials $c$ is negligible. Fine powders, however, are generally cohesive. The linear form of Coulomb's law is applicable to granular materials at relatively high stresses, but in many situations one is dealing with powders at low stresses. The yield locus of cohesive powders shows a marked curvature at negative values of $\sigma$ [16], as depicted in Fig. 1. The point of the yield locus on the negative $\sigma$ axis is called the tensile strength $\sigma_{t}$, which is the maximum tensile stress that the powder can withstand. The tensile strength of the powder is caused by interparticle cohesive forces. Experimental measurements [17] indicate that the tensile strength $\sigma_{t}$ and the cohesion $c$ are of the same order of magnitude. It is also known from experiments $[12,18]$ that the tensile strength increases with the stress under which the material was previously consolidated: $\sigma_{c}$. The angle of internal friction $\phi$ has been related to the frictional behavior at interparticle contacts [19,20]. Experimental works indicate that the angle of internal friction is a density dependent property (see Table 3.1 of Ref. [18]). For example, it has been reported that the value of $\phi$ for wheat raises from $15^{\circ}$ for a powder bulk density $\rho=6.5 \mathrm{kN} / \mathrm{m}^{3}$ up to $30^{\circ}$ for $\rho=9.0 \mathrm{kN} / \mathrm{m}^{3}$.

The next sections are organized as follows. In Sec. II we describe our experimental materials. In Sec. III experimental results for large beads of the same material as our fine toner particles are shown. In Sec. IV the experimental technique and results for fine powders will be described. Finally, conclusions are drawn.

\section{MATERIALS}

We have carried out experiments using a commercially available xerographic toner (Canon CLC500 toner) which flows very well in spite of its small particle size (8.5- $\mu \mathrm{m}$-volume average diameter). The density of the Canon CLC500 toner particles, determined by using an Accu-Pyc 1330 Pycnometer, is $\rho_{p}=1199 \mathrm{~kg} / \mathrm{m}^{3}$, and they are rather monodisperse. Measurements have also been made on three model xerographic toners, designated RT5115, RT5116, and RT5117. These toners are identical in particle size $(12.7-\mu \mathrm{m}$ diameter), particle density $\left(\rho_{p}=1065 \mathrm{~kg} / \mathrm{m}^{3}\right)$, particle resin type (styrene-butadiene), and pigment concentration, but with concentrations of $0.1 \%, 0.2 \%$, and $0.4 \%$ by weight of surface additive, respectively. This additive (Aerosil R812), consists of agglomerates of nanoparticles of fumed silica. From scanning electron microscopy pictures of the toner, we have observed that the individual additive particle has a diameter of $7 \mathrm{~nm}$, and that the additive particles are distributed in agglomerates with estimated diameters from 40 to $60 \mathrm{~nm}$. The effect of Aerosil on the powder tensile strength and packing fraction was described in our previous paper [12]. The addition of these nanoparticles results in a reduction in the powder tensile strength, because the additives are made of a hard material, and therefore they increase the hardness of the contacts; they also reduce the powder tensile strength by reducing the size of the contacts. A higher additive level increases the ability of the particles to rearrange themselves in more packed structures because of the reduction in powder cohesivity. In this paper we investigate the effect of surface additive on the angle of avalanche of these fine cohesive powders. The free volume $\epsilon$ of xerographic toners was measured elsewhere [12-14], and ranges from 0.6 to 0.8 depending on the consolidation stress and powder cohesivity. Particles pack more closely as cohesion decreases and consolidation stress increases. It must be noted that these powders form very open structures as compared to noncohesive granular materials, for which the free volume is of the order of 0.4 [21]. In the case of noncohesive materials the absence of cohesivity allows the particles to roll or slide easily over one another. However, interparticle adhesive forces in fine cohesive powders oppose gravity and particle restructuring, leading to large values of the free volume. In the strong cohesive limit particles build up columnar structures containing a large number of voids, giving a free volume close to the random ballistic deposition limit ( $\epsilon$ $\simeq 0.87$ ) [21].

To make clear the fundamental role of particle size in the avalanches of granular materials we have carried out experiments with polymer beads (XP808 beads) which is a low cohesive granular material. This material consists of a mixture of spherical beads of styrene butadiene (same resin as toners), with $40 \%$ by weight in the size range of 500-300$\mu \mathrm{m}$ diameter; $35 \%$ in the range $300-210 \mu \mathrm{m} ; 12 \%$ in the range $210-106 \mu \mathrm{m} ; 8 \%$ in the range $106-53 \mu \mathrm{m}$; and the remainder, about $5 \%$, below $53 \mu \mathrm{m}$.

\section{AVALANCHES AND ANGLE OF REPOSE OF NONCOHESIVE GRANULAR MATERIALS}

There is an upper limit to the slope that a granular material can sustain, and beyond this limit a slip surface forms and the material avalanches to produce a lower slope. The maximum stable angle is related to the angle of internal friction $\phi$, but the relationship depends on the cohesivity of the material [see Eq. (2)]. In the noncohesive limit the force 
TABLE I. Angle of fracture $\theta$ for XP808 beads as a function of bed width $D$.

\begin{tabular}{cccccc}
\hline \hline$D(\mathrm{~cm})$ & 10.00 & 4.35 & 3.15 & 2.05 & 1.50 \\
\hline$\theta(\mathrm{deg})$ & $13.7 \pm 0.6$ & $15.1 \pm 1.0$ & $14.4 \pm 0.6$ & $16.6 \pm 2.3$ & $22.9 \pm 1.7$ \\
\hline \hline
\end{tabular}

acting on an incipient slip plane is simply, $F=\mu N$, where $N$ is the normal force and $\mu$ is the coefficient of internal friction, which is related to the angle of internal friction $\phi$ by $\tan \phi=\mu=F / N$; it then follows that the maximum angle in the absence of failure in an ideal cohesionless material is equal to the angle of internal friction. The length of the slope does not enter into this calculation, so the maximum stable angle in the noncohesive limit is independent of the length of the slope. The angle of repose of a conical pile of a noncohesive material, and similarly the angle of a conical hollow formed in a bunker in the core flow, are slightly lower than the maximum stable angle. Nevertheless, for noncohesive materials the angle of repose is very close to the angle of failure, and is commonly used as an estimate of the angle of internal friction. Clearly, however, this cannot be applied to fine cohesive powders such as xerographic toner. We conducted experiments to measure the angle of internal friction of the mixture of noncohesive XP808 beads described in Sec. II, but when we came to measure this angle the results depended on the experiment.

(i) Pouring the beads into a dish and measuring the angle of repose gave values in the interval from $24^{\circ}$ to $27^{\circ}$. This range fits the range of values found in literature for smooth noncohesive particles [6].

(ii) Dry nitrogen was passed through the granular bed, and the material expanded to an average free volume of $\simeq 0.49$. Then the gas flow was turned off and the material was allowed to settle. The settled free volume was $\simeq 0.45$, which corresponds to a loose packed bed of uniform, noncohesive spheres [21]. We slowly tilted the initialized bed, and obtained the results for the maximum stable angle shown in Table I. We note that with the exception of the $1.5-\mathrm{cm}$ bed results, which are anomalously high due to wall effects, the maximum stable angle fall within the range $\alpha=14.9 \pm 1.6^{\circ}$; thus the angle of internal friction for this dilated bed is only $60 \%$ of the angle of repose of the poured cone.

(iii) The material was initialized as in (ii), and then the bed was tapped by hand to promote maximum rearrangement of the material. This leaded to a decrease in the free volume down to $\simeq 0.39$, which corresponds to a dense packed bed of uniform, noncohesive spheres. Then, as in (ii), the bed was tilted to measure its maximum stable angle giving the results shown in Table II.

In all cases the avalanches observed consisted of a superficial layer of several grains. As may be seen from Tables I and II, the maximum stable angle depends significantly on

TABLE II. Angle of fracture $\theta$ for XP808 beads as a function of the bed width $D$ previously tapped.

\begin{tabular}{cccccc}
\hline \hline$D(\mathrm{~cm})$ & 11.7 & 8.5 & 6.4 & 5.2 & 4.1 \\
\hline$\theta(\mathrm{deg})$ & $21.8 \pm 2.0$ & $27.6 \pm 2.3$ & $28.8 \pm 1.5$ & $30.2 \pm 0.6$ & $31.0 \pm 1.3$ \\
\hline \hline
\end{tabular}

bed diameter in case (iii) but not in case (ii). Presumably the reason for this behavior would be the effect of the walls on the stress distribution within the dense packed bed. Summing over all five bed diameters, the average value of the angle of internal friction for the dense packed bed is $27.9 \pm 3.9^{\circ}$; this is about twice the nonrearranged angle of internal friction. This result suggests that the angle of internal friction depends strongly on the packing fraction of the material as already found in the literature for other granular materials [18]. On the other hand, the value for the angle of internal friction of the tapped bed is close to the measured angle of repose of a poured, conical pile. This indicates that when beads are poured, forming a cone, the particles are subjected to collisions that eliminate relatively unstable configurations and pack near the limit of complete restructuring, resulting in an angle of repose similar to the angle of internal friction of the rearranged bed. We conclude from these results that the rearrangement of granular materials plays an important role in determining the angle of internal friction, and that a selfconsistent theory of shear fracture of noncohesive materials must include the effect of rearrangement.

\section{AVALANCHES IN FINE COHESIVE POWDERS: EXPERIMENTAL TECHNIQUE AND RESULTS}

The yield locus of a fine cohesive powder is very sensitive to its degree of consolidation, and in order to obtain reproducible results it is necessary to achieve a consistent, initial state. To achieve this the powder was fluidized by a stream of dry nitrogen, so that the material became quite uniform, the fluidizing gas was then turned off, and the powder settled under its own weight to a static height into a reproducible consolidated state. All our measurements began with this initialization step, so that the particles were dried and in a reproducible state of consolidation. The next step was to tilt the bed, increasing the angle from zero until the powder avalanched. To eliminate vibrations in the tilt drive we installed a multiple gear train, driven by a dc electric motor. This enabled us to adjust the tilt speed in the range 0.2-1 rad per minute, and eliminate the vibrations. As the angle of tilt increased, a shear stress was generated in the powder layer. There came a point at which the sample failed in shear and a layer of powder slumped to one side of the bed. The problem then was to relate the angle of avalanche to the stresses in the slip plane. We then measured the thickness of the sheared layer $\left(h_{s}\right)$ and the tilt angle at which powder avalanched $(\alpha)$ as functions of bed width and sample depth. We considered the effect of the bed width on avalanches in order to investigate the constraints imposed by the retaining walls.

The problem of estimating the avalanche depth is related to the problem of measuring the profile of the post-avalanche powder surface. The technique we developed makes use of the tendency of powder particles to adhere to a paper surface: a piece of paper card of the same width as the bed diameter was lowered edgewise into the powder surface, so as to cleave the powder; then, after carefully withdrawing the card, the card was found to carry a replica of the powder profile. Examples of such profiles are shown in Figs. 2 and 3. These profiles give the location of the slip surface, though some assumptions have to be made near the lower wall, as will be shown. We have also used a video camera which 


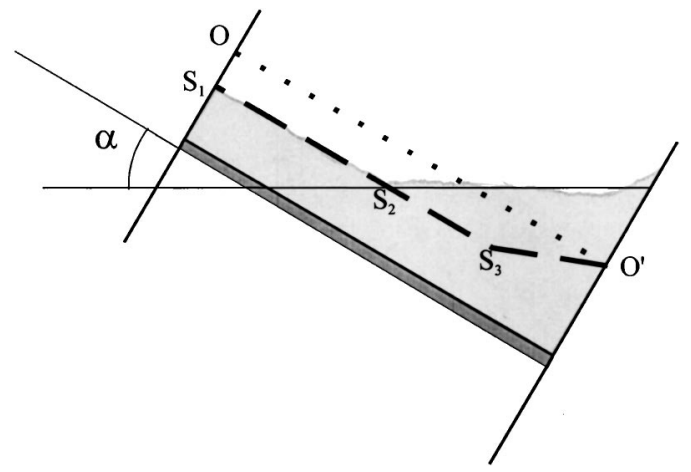

FIG. 2. Example of the powder free surface profile after the avalanche $(D=5.17 \mathrm{~cm}$, toner Canon CLC500). The free surface before the avalanche $(\cdots)$ and the slip planes computed by using the model of wedges $(---)$ are shown.

recorded the evolution of the material at a rate of 25 frames per second. The camera was connected to a computer for image processing.

\section{A. Profile of the slip surface}

In these experiments, we used cylindrical beds of several diameters, and rectangular beds of several widths in order to investigate the importance of the wall effect in the onset of flow. In order to understand the onset of avalanches in fine cohesive powders, we need to have accurate values of the avalanche angle and the location and shape of the slip surface. With the experimental techniques described above, reliable values of $\alpha$ were obtained, but information about the slip surface has to be inferred from the post-avalanche surface profile. In narrow beds, post-avalanche surface profiles (such as shown in Fig. 2) took the form of pairs of intersecting planes. In the case of rectangular beds it is then a matter of simple geometry to calculate the position of the initial powder surface, designated $00^{\prime}$ in Fig. 2. The slip surface indicated in Fig. 2 is partly a matter of conjecture: one section of the slip surface $\left(S_{1} S_{2}\right)$ was exposed when the surface layer slides away, but the remaining section lied hidden; however we can locate the two terminal points of the hidden section: (i) the slip surface is pinned at the intersection of the

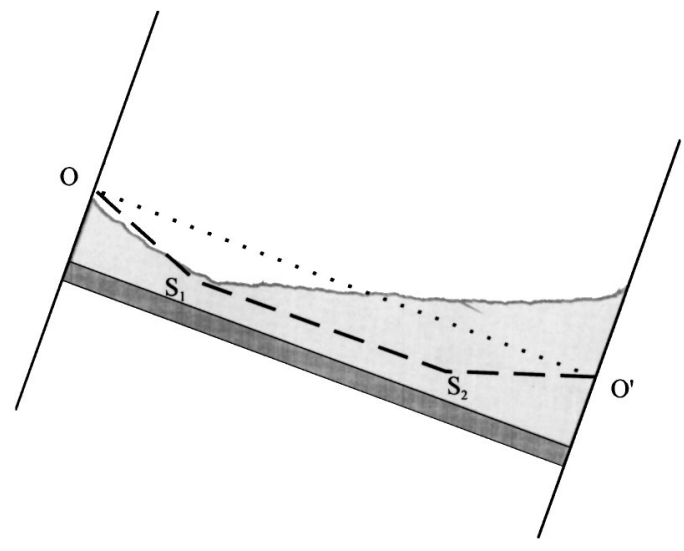

FIG. 3. Example of the powder free surface profile after the avalanche $(D=11.7 \mathrm{~cm}$, toner Canon CLC500). The free surface before the avalanche $(\cdots)$ and the slip planes computed by using the model of wedges $(---)$ are shown.

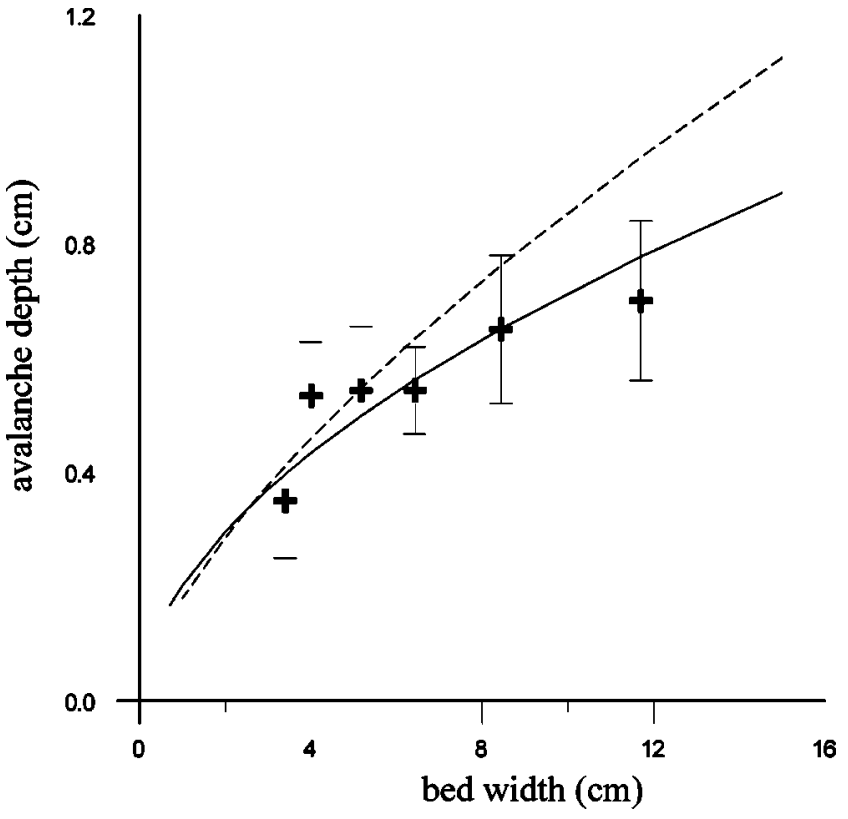

FIG. 4. Experimental values of avalanche depth $h_{s}$ vs bed width $D$ for toner Canon CLC500. Computed theoretical curves according to the two-slip-plane model (- - ) of Sec. V C and the three-slipplane model (- - -) of Sec. V D are shown.

initial powder surface and the lower retaining wall (point $O^{\prime}$ in Fig. 2); and (ii) the exposed and buried sections of the slip surface must meet at point $S_{2}$. The buried section of the slip surface may therefore be approximated by line segments $S_{2} S_{3}$ and $S_{3} O^{\prime}$, though the exact location of the slip surface is not known a priori. For large beds $(D>8 \mathrm{~cm})$ a small curvature of the slip surface was observed in its upper corner, as shown in the experimental post-avalanche surface profile depicted in Fig. 3. It is remarkable that the depth of avalanches (several millimeters) is very large compared to the particle diameter, and depends on the separation of the retaining walls. This is an effect of the huge cohesion existing between particles as compared to their weight. Definitely, avalanches in fine cohesive powders are qualitatively different to avalanches in noncohesive or slight-cohesive granular media.

An interesting observation to note is that analyzing the avalanche by video camera and recorder we found that the powder became fluidized during the avalanche. Fluidization occurred if the velocity of the avalanche exceeded a certain critical value depending on powder cohesivity. The fluidized powder took the flow properties of a low viscosity fluid, moving under the influence of gravity, so that, when the layer came to rest and settled, its upper surface was horizontal (as shown in Figs. 2 and 3). As predicted [22], the inertial regime did not exist for this fine powder.

\section{B. Avalanche angle and avalanche depth}

We measured the maximum depth of the slip plane $h_{s}$ (avalanche depth) and the angle of avalanche $\alpha$. Results of these tests for the Canon CLC toner are plotted in Figs. 4 and 5. We found an increase in $h_{s}$ with bed width, as shown in Fig. 4. Also the angle of avalanche decreased with increasing bed width, as shown in Fig. 5. We note that in the range of very narrow beds the variation of the avalanche angle is very 

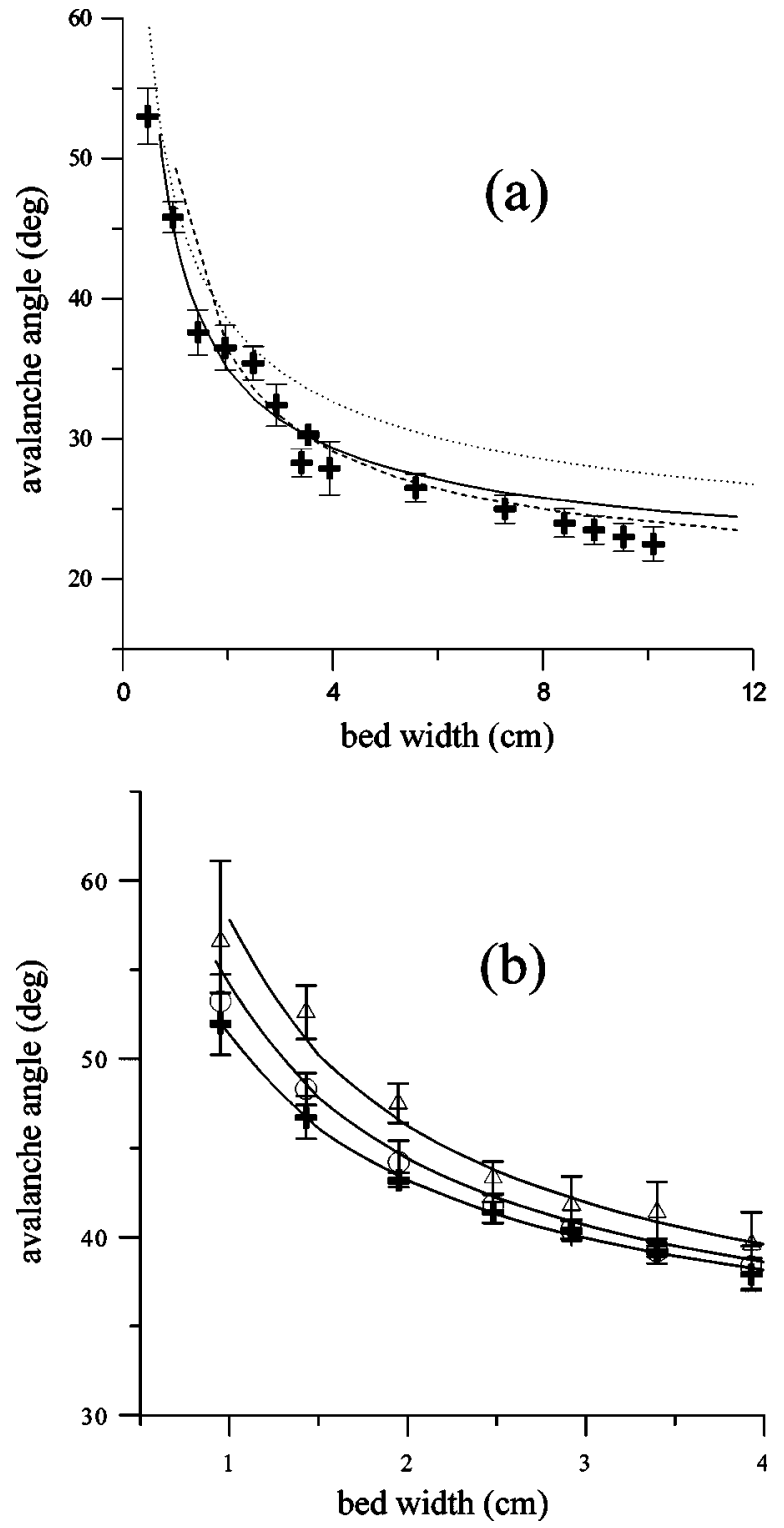

FIG. 5. (a) Experimental values of the angle of avalanche, $\alpha$, vs bed width $D$, for toner Canon CLC500. Computed theoretical curves are shown: Triangular model $(\cdots)$, two-slip-plane model (- $)$, and three-slip-plane model ( ---$)$. (b). Experimental values of the angle of avalanche, $\alpha$, vs bed width $D$, for a series RT of model toners. $\triangle: 0.1 \%$ by weight of surface additive. $\bigcirc: 0.2 \%$ by weight of surface additive. $+: 0.4 \%$ by weight of surface additive. Computed theoretical curves from the two-slip-plane model for each data series are shown.

pronounced, while it decreases smoothly in the range of large beds. We made the experiment with a 40-cm-width bed, and the avalanche angle was $19^{\circ}$ clearly below the values obtained for slightly or noncohesive smooth particles [6], and also below the angle of repose obtained for spherical noncohesive polymer beads (see Sec. III). In the limit $D$ $\rightarrow \infty$ it is expected that the angle of avalanche will approach the angle of internal friction. Also, there is a critical value of the bed width (around $0.5 \mathrm{~cm}$ ) below which the powder can sustain $90^{\circ}$ slopes. In Fig. 5(b) results of the angle of avalanche for the model toners are shown in the range of bed widths where the strongest variation occur. We note that there is a small decrease in $\alpha$ with the surface additive per-

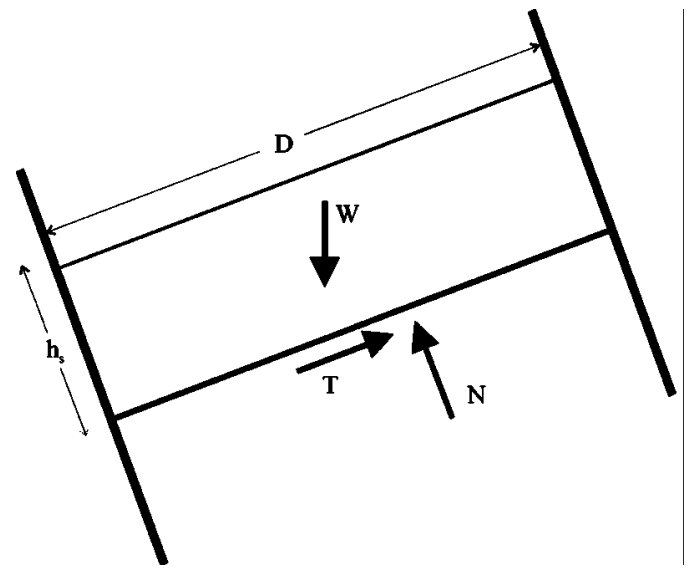

FIG. 6. Planar wedge model. The fracture surface is assumed to be a plane parallel to the free surface. Normal and tangential stresses acting on the slip surface are represented.

centage, as we would expect for lower values of cohesivity, though the trend is not marked. In contrast, the angle of avalanche for the Canon toner is about $10^{\circ}$ lower than for the $\mathrm{RT}$ series over much of the range.

On the other hand, these tests showed that the avalanche angle $\alpha$ and the characteristic avalanche depth $h_{s}$ were not affected by the sample depth, provided the bed height was larger than the avalanche depth.

\section{THEORETICAL ANALYSIS: METHOD OF WEDGES}

We now turn to the theoretical analysis of the slip problem, based on Coulomb's method of wedges [18,23,24]. Several models are considered, starting with a simple wedge that corresponds to an ideal, long slope; we then build up to compound wedges to take account of the wall effect in beds of finite width.

This method of analysis dates back to Coulomb's pioneering work on soil mechanics in 1773 [15]. The underlying assumptions in the method of wedges are (i) that failure occurs along a slip surface which is planar, (ii) that the stresses at the slip surface are equal to the values specified by the yield locus, and (iii) that the condition of critical equilibrium holds (i.e., just before the avalanche, we can assume a balance of forces in the sliding wedge). The method is commonly applicable to two-dimensional situations, such as the calculation of the force on an infinite retaining wall [18]. One of the standard applications of the method of wedges [18] is the analysis by geologists of hill slope, as when topographically induced gravitational shear stress exceeds the strength of the material and a landslide occurs [23]. In this work we have considered four cases, increasing in complexity, each depending on an assumed form of the slip surface.

(i) A slab of uniform thickness where the slip surface is a plane, parallel to the top of a sample, as shown in Fig. 6. In this case the effect of the walls is not included in the analysis.

(ii) A simple wedge, as in Fig. 7. In this case the angle of the slip plane $\beta$ and the width of the bed determine the geometry of the wedge. [Note that the slip planes in Fig. 2 are between cases (i) and (ii).] 


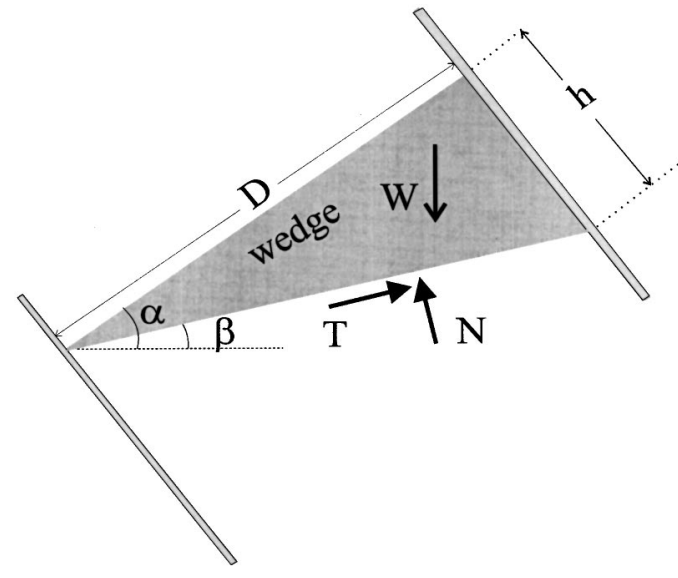

FIG. 7. Triangular wedge model. The fracture surface is assumed to be a plane inclined at an angle $\beta<\alpha$. Normal and tangential stresses acting on the slip surface are represented.

(iii) and (iv) Composite wedges corresponding to intersecting slip planes, as shown in Figs. 8 and 9. The effect of the lower retaining wall is implicit in the calculation (iii), and both upper and lower walls are included in (iv).

\section{A. Planar wedge model}

In this, the simplest case, we assume that the slip surface is plane, parallel to the initial, unperturbed surface of the powder. The condition of critical equilibrium is given by Eq. (2). Neglecting the effect of the walls, the shear and compressive stress are

$$
\begin{gathered}
\tau=\left[1-\epsilon\left(h_{s}\right)\right] \rho_{p} g h_{s} \sin \alpha, \\
\sigma=\left[1-\epsilon\left(h_{s}\right)\right] \rho_{p} g h_{s} \cos \alpha .
\end{gathered}
$$

Here $\alpha$ is the angle of tilt, $h_{s}$ is the depth of the slip plane, $\epsilon\left(h_{s}\right)$ is the free volume at the slip plane, and $\rho_{p}$ is the particle density. Tilted bed measurements $[16,17]$ indicate that cohesion is close in magnitude to the tensile strength $\sigma_{t}$. Therefore, we substitute $\sigma_{t}\left(h_{s}\right)$ for $c\left(h_{s}\right)$ in Eq. (2). Hence the angle of internal friction is, approximately,

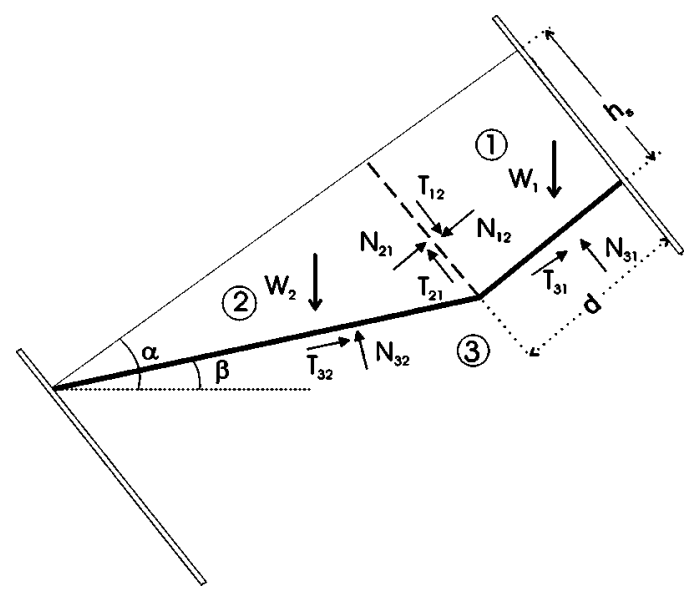

FIG. 8. Composite wedge. The fracture surface is assumed to be composed of two planes, one parallel to the free surface and the other inclined at an angle $\beta<\alpha$. Normal and tangential stresses acting on the slip surfaces are represented.

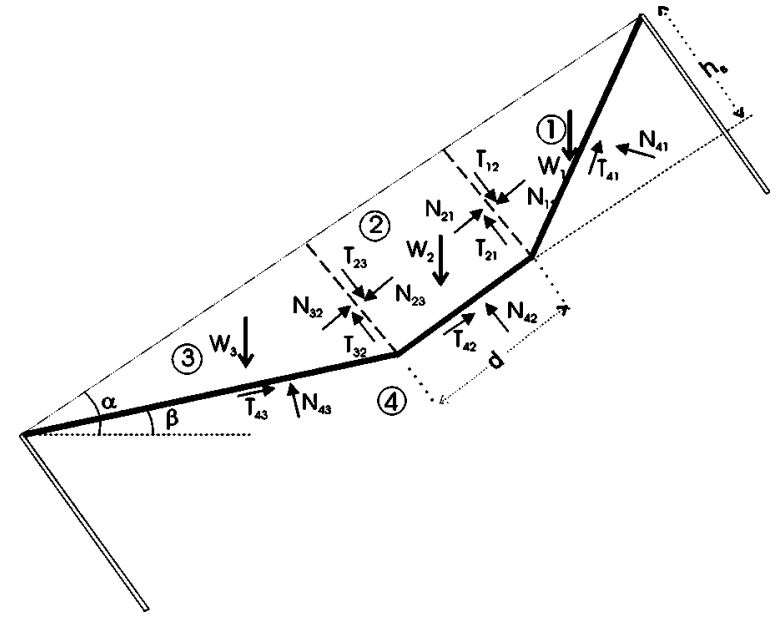

FIG. 9. Composite wedge. The fracture surface is assumed to be composed of three planes. Normal and tangential stresses acting on the slip surfaces are represented.

$$
\tan \phi=\frac{\tau-c\left(h_{s}\right)}{\sigma} \approx \frac{(1-\epsilon) \rho_{p} g h_{s} \sin \alpha-\sigma_{t}\left(h_{s}\right)}{(1-\epsilon) \rho_{p} g h_{s} \cos \alpha} .
$$

To illustrate this method of analysis we use it to calculate the angle of internal friction of Canon CLC500 cyan toner for a range of bed widths, $D$. Values for $\sigma_{t}$ and $\epsilon$ versus depth for this toner were already measured [12]. The calculation of $\phi$ then follows from equation 5 using the measured values of $h_{s}$ and $\alpha$. Note that as the model does not take account of the wall effect we should therefore see a trend in $\phi$ with $D$. The results are summarized in Table III. Here we see the expected variation in $\phi$ with bed width. At large values of bed width, for which the wall effect are expected to be less important, $\phi=10^{\circ}$. This result is consistent with the measured slope of the yield locus of this low cohesive toner [17]. The reason for such a small value of $\phi$ can be attributed to the high free volume of the toner $(\sim 0.7)$ which gives a low value for powder bulk density $\left(\rho \sim 360 \mathrm{~kg} / \mathrm{m}^{3}\right)$. The anomalously high values of $\phi$ at small bed widths is the result of neglecting the retaining effect of the walls. Clearly the wall effect needs to be taken into account, and to do this we introduce more elaborate wedge models.

\section{B. Triangular wedge model}

The geometric arrangement of Fig. 7 shows a hypothetical slip plane. From Fig. 7, wedge equilibrium equations

$$
\begin{gathered}
T \cos \beta-N \sin \beta=0, \\
T \sin \beta+N \cos \beta=W,
\end{gathered}
$$

and a critical equilibrium equation

TABLE III. Apparent angle of internal friction of Canon CLC500 toner according to the planar wedge model.

\begin{tabular}{lccccc}
\hline \hline$D(\mathrm{~cm})$ & 11.7 & 8.5 & 6.4 & 5.2 & 4.1 \\
\hline$\phi(\mathrm{deg})$ & 10.3 & 12.2 & 12.4 & 17.6 & 19.1 \\
\hline \hline
\end{tabular}




$$
T=N \tan \phi+c \frac{D}{\cos (\alpha-\beta)}
$$

follow, where $T$ and $N$ are the tangential and normal forces, respectively, over the slip plane, $W=\frac{1}{2} \rho_{p}(1-\epsilon) g D^{2} \tan (\alpha$ $-\beta)$ is the wedge weight, and $D / \cos (\alpha-\beta)$ is the length of the slip plane. Equations (6), (7), and (8) must be satisfied with two unknowns $T$ and $N$. A slip plane appears when the set of three equations is compatible. The condition for compatibility gives a functional relation $\alpha=\alpha(\beta)$ between the angle of tilt $\alpha$, and the angle $\beta$. The function $\alpha(\beta)$ has an absolute minimum $\alpha_{\text {min }}$ at a given value of $\beta_{0}$. Now, if we slowly tilt the bed, a slip plane is not formed until the angle $\alpha_{\min }$ is reached. Once this angle is reached the powder avalanches, and the value of $\beta_{0}$ determines the avalanche depth. Operating, we obtain

$$
\begin{gathered}
\beta_{0}=\frac{\alpha+\phi}{2}, \\
\rho_{p}(1-\epsilon) g D=4 C \cos \phi[1-\cos (\alpha-\phi)]^{-1} .
\end{gathered}
$$

If we approximate $1-\cos (\alpha-\phi) \approx(\alpha-\phi)^{2} / 2$, substitute in Eq. (10), and rearrange, we have

$$
\alpha=\phi+\left[\frac{8 c}{\rho_{p}(1-\epsilon) g D} \cos \phi\right]^{1 / 2} .
$$

From Eq. (11) it is apparent the key role played by powder cohesion $c$ and bed width $D$ on the onset of avalanches. In the noncohesive limit, $c \rightarrow 0$ and $\alpha \rightarrow \phi$, as expected. From Eq. (11) we see that avalanche angle $\alpha$ should be linearly related to $D^{-1 / 2}$, with the intercept $\alpha=\phi$ at $D \rightarrow \infty$, where the wall effect is negligible. Also, there is a limit value of $D$ for which $\alpha=90^{\circ}$; for narrower beds the powder does not avalanche. Two important limitations to analyze our experimental results are that cohesion and free volume $\epsilon$ are not constant with depth for a fine cohesive powder. Experimental measurements on this toner [12] show that powder free volume does not vary considerably in the range of values measured of the avalanche depth (Fig. 4), and based on this we can assume a constant average value of free volume. Conversely, the variation rate of the tensile strength is very large. In Fig. 10 we represent the measured tensile strength $\sigma_{t}$ for toner Canon CLC500 as a function of the powder depth $h$ measured from the free surface. These measurements were made using a powder bed technique [12], and show a marked linear increase of the tensile strength with depth, $\sigma_{t}=\sigma_{t 0}+\delta h$. Clearly this variation has to be taken into account in the wedge model. Therefore, the average value of cohesion $\langle c\rangle$ along the slip plane has to be used in Eq. (8). As cohesion and tensile strength are comparable:

$$
\langle c\rangle \simeq \sigma_{t 0}+\delta \frac{h}{2}=\sigma_{t 0}+\frac{D \tan (\alpha-\beta)}{2} .
$$

Solving for $T$ and $N$ in Eqs. (6) and (7), and substituting in Eq. (8), we obtain,

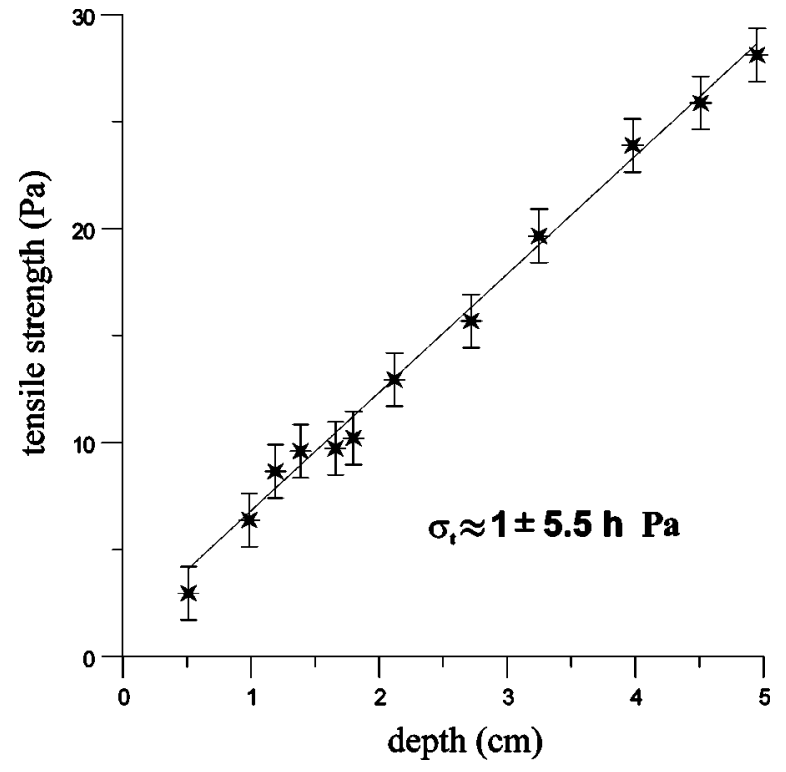

FIG. 10. Measured tensile strength versus depth for toner Canon CLC500. The line represents the least squares fit to the experimental data.

$$
\begin{aligned}
\frac{\rho_{p} g(1-\epsilon) D}{2 \cos \phi}= & \frac{\sigma_{t 0}}{\sin (\alpha-\beta) \sin (\beta-\phi)} \\
& +\frac{\delta D}{2 \cos (\alpha-\beta) \sin (\beta-\phi)} .
\end{aligned}
$$

The minimum of $\alpha$ is found when

$$
\frac{2 \sigma_{t 0}}{\delta D}=\tan ^{2}(\alpha-\beta) \cot (2 \beta-\phi-\alpha) .
$$

In the case of constant cohesion ( $\delta=0$ ) we recover Eq. (9). Equations (13) and (14) determine the theoretical angle of avalanche. In the asymptotic limit $D \rightarrow \infty$, from Eq. (14), $\beta$ $\rightarrow \alpha$, and, from Eq. (13) $\alpha \rightarrow \phi+\arcsin \left[\delta \cos \phi /\left(\rho_{p}(1\right.\right.$ $-\epsilon) g)]$. This result would imply that in the asymptotic limit of very wide beds the powder would avalanche at a given angle larger than the angle of internal friction. However, for very large values of $D$ and the avalanche depth, the average consolidation of the powder would be very large; experiments show that for high consolidations the rate of increase of the tensile strength is sublinear $[13,14], \sigma_{t}=a h^{b}(b$ $<1$ ). It can be checked that, using this sublineal law for the cohesion in the triangular wedge model, $\alpha \rightarrow \phi$ when $D$ $\rightarrow \infty$.

We have used the triangular model results to analyze our experimental measurements of the avalanche angle of the Canon CLC500. The value of $\sigma_{t 0}$, the tensile strength at zero depth, cannot be obtained directly by extrapolation of $\sigma_{t}$ to zero depth, because the errors in our measurements are comparable to the magnitude of $\sigma_{t 0}$ but $\sigma_{t 0}$ is about $1 \mathrm{~Pa}$ in order of magnitude. In contrast to $\sigma_{t 0}$, the slope $\delta$ is well characterized for this toner, $\sigma_{t} \simeq 1+5.5 h \mathrm{~Pa}$, where $h$ is in centimeters (see Fig. 10). In accordance with experimental measurements [12], we have taken an average value of 0.7 for powder free volume $\epsilon$. The only property for which we do not have prior information is the angle of internal friction, $\phi$. 
We used the value of $\phi=10^{\circ}$ for the Canon CLC500 toner, resulting from calculations based on the planar wedge model in the limit of large beds and from direct measurements of the slope of the yield locus [17]. In Fig. 5 theoretical and experimental results for the avalanche angle are shown. As can be seen, the prediction of the model is good only for narrow beds. This result makes sense, as the triangular wedge is not appropriate to model the slip plane for wide beds, where the inferred profile of the slip surface is quite distinct from what is assumed in the triangular model. In the next two sections we will consider more realistic wedge models according to slip surface profiles inferred from experimental observations.

\section{Two-slip-plane model}

As we have seen, for beds of width less than about $8 \mathrm{~cm}$, the post-avalanche surface profiles can be approximated by a pair of intersecting planes, and this leads to the conclusion that the corresponding slip surfaces can also be approximated by two intersecting planes, as shown in Fig. 2. In this section we summarize the steps taken in an alternative approach to the triangular wedge model, involving an analysis of a composite wedge.

To apply the critical equilibrium condition the composite wedge is divided into two blocks, as shown in Fig. 8, where the wedge profile is specified by the angle $\beta$ and the distance $d$. Stresses in the composite layer are such that when the wedge slips there must be a slip line propagating from the corner between regions 1 and 2, shown as a dashed line in Fig. 8 [24]. There is no criterion to locate this slip line, but our final results are almost independent of the orientation of the line, and we have therefore chosen to locate it in the most convenient position for our calculation. On each block of the wedge the condition of static equilibrium must apply (i.e., the sum of forces must equal zero). Also, along each slip surface the Coulomb limit equilibrium must be satisfied. From this we have a total of nine equations:

$$
\begin{gathered}
T_{31} \cos \alpha-N_{31} \sin \alpha+N_{21} \cos \alpha-T_{21} \sin \alpha=0, \\
T_{31} \sin \alpha+N_{31} \cos \alpha+N_{21} \sin \alpha+T_{21} \cos \alpha=W_{1}, \\
T_{32} \cos \beta-N_{32} \sin \beta-N_{12} \cos \alpha+T_{12} \sin \alpha=0, \\
T_{32} \sin \beta+N_{32} \cos \beta-N_{12} \sin \alpha-T_{12} \cos \alpha=W_{2}, \\
T_{12}=T_{21}, \\
N_{12}=N_{21}, \\
T_{31}=N_{31} \tan \phi+\left\langle c L_{31}\right\rangle, \\
T_{32}=N_{32} \tan \phi+\left\langle c L_{32}\right\rangle, \\
T_{12}=N_{12} \tan \phi+\left\langle c L_{12}\right\rangle,
\end{gathered}
$$

where $L_{i j}$ is the length of the slip plane dividing blocks $i$ and $j ; T_{i j}$ and $N_{i j}$ represent tangential and normal forces, respectively, over the slip planes, as indicated in Fig. 8; and $W_{i}$ is the weight of block $i$. Equations (15)-(23) must be satisfied with eight unknowns $T_{12}, T_{21}, N_{12}, N_{21}, T_{32}, N_{32}, T_{31}$,
TABLE IV. Measured average free volume $\langle\epsilon\rangle$ and rate of increase of the tensile strength $\sigma_{t}$ with depth for the RT series of model toners.

\begin{tabular}{lccc}
\hline \hline Toner & Aerosil $(\%)$ & $d \sigma_{t} / d h(\mathrm{~Pa} / \mathrm{cm})$ & $\langle\epsilon\rangle$ \\
\hline RT5115 & 0.1 & 9.2 & 0.674 \\
RT5116 & 0.2 & 6.5 & 0.642 \\
RT5117 & 0.4 & 3.6 & 0.624 \\
\hline \hline
\end{tabular}

and $N_{31}$. The condition of compatibility of these equations yields a functional relation $\alpha=\alpha(\beta, d)$ between the angle of tilt $\alpha$, and the variables $\beta$ and $d$. The function $\alpha(\beta, d)$ presents a minimum at given values of $\beta_{0}$ and $d_{0}$. Once the minimum angle, making possible critical equilibrium, is reached, the powder avalanches. Then we must find the minimum value of $\alpha$ which is solution of critical equilibrium equations. The values of $\beta_{0}$ and $d_{0}$ for which this minimum occurs determine the theoretical avalanche depth $h_{s}$. In Figs. 4 and 5 the resulting theoretical curves of $h_{s}$ versus $D$ and $\alpha$ versus $D$ are presented, together with the experimental results for the Canon CLC500 toner. Again for theoretical calculations we used the value of $\phi=10^{\circ}$ resulting from previous calculations and measurements. As can be seen from Fig. 5 , a better agreement between experimental and theoretical results is obtained with this composite wedge model.

In agreement with experiments, the theory also indicates that there is a critical value of bed width of about $0.5 \mathrm{~cm}$, below which the bed cannot avalanche at any inclination. This corresponds to the wall effect on cohesive powders inferred from our other measurements and calculations. It is, of course, the wall effect that provides the reason for the composite wedge, as shown in Fig. 8. If now we take the various results from the analysis, compute the form of the wedge, and compare it with a typical post-avalanche profile, we obtain the results shown in Fig. 2 for Canon CLC500 toner in a 5.17-cm-diameter bed. As can be seen, the theoretical slip plane is in reasonable agreement with the observed postavalanche profile.

We have used this wedge model to calculate the avalanche characteristics and angle of internal friction for the RT series of model xerographic toners (RT5115, RT5116, and RT5117, with $0.1 \%, 0.2 \%$, and $0.4 \%$ Aerosil, respectively). For these calculations we need to know the tensile strength $\sigma_{t}$, the bulk density, and the dependence of free volume, $\epsilon$, on depth in the bed. From our previous work [12] we can assume that $\sigma_{t 0}$ is about $1 \mathrm{~Pa}$ for the three model toners. In contrast to $\sigma_{t 0}$, the slopes $d \sigma_{t} / d h$ are well characterized for these toners, and the values taken from our previous paper [12] are shown in Table IV, together with values of mean free volume $\langle\epsilon\rangle$ calculated for a consolidation stress of $100 \mathrm{~Pa}$ (i.e., approximately $1 \mathrm{~g} / \mathrm{cm}^{2}$ ). In Fig. 5(b) the resulting theoretical curves of $\alpha$ versus $D$ are presented, together with the experimental results. The best fits to the data are obtained using the values of $\phi$ shown in Table $\mathrm{V}$. These results indicate that the higher percentage Aerosil (which is associated to a higher surface rugosity) the higher values of the angle of internal friction. Using the measured values of the mean free volume $\langle\epsilon\rangle$ and the particle density $\rho_{p}$ for each toner, we have calculated the toner bulk density $\rho=\rho_{p}(1-\langle\epsilon\rangle)$, and represented the obtained angle of inter- 
TABLE V. Calculated values of the angle of internal friction for the RT series of model toners according to the two-slip-plane wedge model.

\begin{tabular}{lccc}
\hline \hline Toner & RT5117 & RT5116 & RT5115 \\
\hline$\%$ Aerosil & 0.4 & 0.2 & 0.1 \\
$\phi\left(^{\circ}\right)$ & 23 & 15 & 8 \\
\hline \hline
\end{tabular}

nal friction as a function of $\rho$ (see Fig. 11). From this figure it is clear the increase of $\phi$ with the powder bulk density.

\section{Three-slip-plane model}

For large beds we need to take account of the upper wall, in addition to the lower; in Fig. 3, for example, there is a distinct curve to the slip surface originating from the upper wall. In order to accommodate this in the theory we have to add a third block to the wedge, so arranged that the upper corner remains pinned to the upper wall (see Fig. 9). As in the case of two slip planes, sharp corners are included between the slip planes, so that a new decomposition of the wedge is needed and we have a total of three blocks composing the theoretical wedge on which critical equilibrium condition must be applied. Note that we now have three independent variables $(\beta, d$, and $h$ ) which determine the wedge geometry for a given $\alpha$. Proceeding in a similar way to the previous calculation we arrive at a compatibility condition for critical equilibrium $\alpha=\alpha(\beta, d, h)$. The first value of $\alpha$ at which this condition is fulfilled is the angle at which the powder avalanches. Therefore, we have to minimize the function $\alpha=\alpha(\beta, d, h)$ in order to find the angle of avalanche.

The theoretical values of $\alpha$ and $h_{s}$ from the three-block model are not very different from the corresponding twoblock model results (see Fig. 5), but the fit to experimental results of the avalanche angle for very large beds is im-

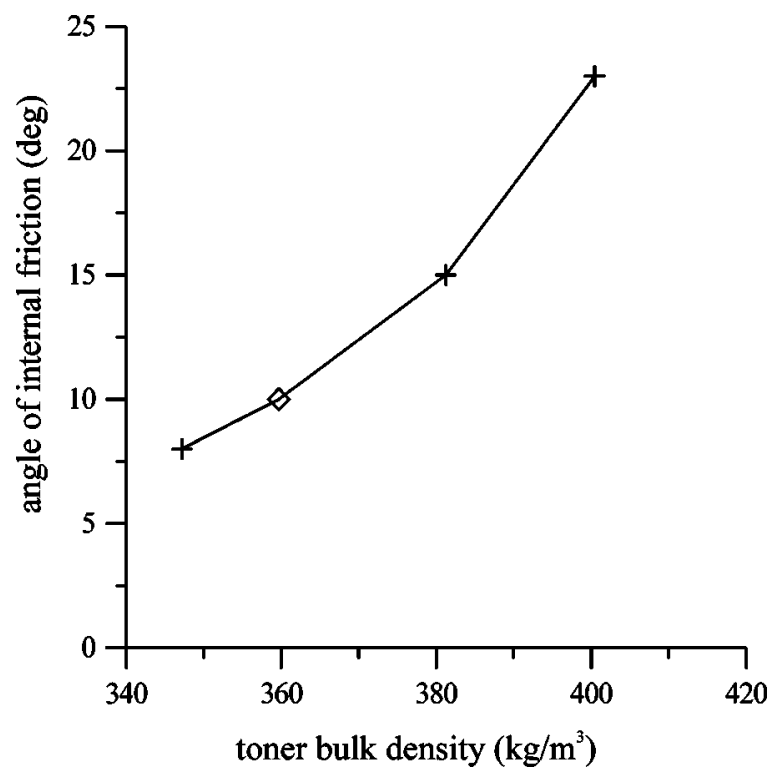

FIG. 11. Calculated values of the angle of internal friction $\phi$ as a function of the measured toner bulk density $\rho .+$ : RT series of model toners. $\diamond:$ Canon CLC500 toner. proved. On the other hand, the slip surface profiles are qualitatively different. In Fig. 3 we present the computed theoretical wedge for the 11.7-cm-diameter bed superimposed on an experimental profile of the powder surface after avalanche. Note that the pinning of the upper extreme of the powder is not perfect; this is due to the fact that as the bed is tilted the powder rearranges due to the shear stress. This may be the origin of the minor discrepancy between the experimental and theoretical values of $h_{s}$ for this model.

\section{SUMMARY AND CONCLUSIONS}

Experimental measurements of the angle of avalanche $\alpha$ of fine cohesive powders reveal that it depends strongly on the width of the bed, especially in the range of narrow beds where $\alpha$ can attain even $90^{\circ}$ for very narrow beds. Also, the avalanche depth depends on the bed width and is of the order of several millimeters, very large compared to particle size. This observation is in contrast with the phenomenon observed in noncohesive large grains, where motion occurs in relatively thin boundary layers of about ten grains, and the stability of slopes is analyzed based on frictional forces between individual grains. Typically, interparticle cohesive forces in fine cohesive powders are very large compared to the particle weight. This characteristic has an essential effect on the stability of slopes, and makes fine powder more akin to wet sand than to dry sand. The forces between particles at the slip plane in fine cohesive powders depend on the stresses exerted by the rest of material and container walls. Therefore, we must consider the influence of boundary conditions and macroscopic stresses, and for this reason we chose a continuum approach to analyze the problem.

From the post-avalanche surface profiles we have deduced that the slip surfaces can be approximated by pairs of intersecting planes, and based on this we have used Coulomb's theory of wedges to analyze our results on a set of fine cohesive powder (xerographic toners). A triangular wedge analysis indicates that the tilt angle $\alpha$ at which avalanche occurs follows a simple relationship with respect to bed width and sample cohesion $\left[\alpha \approx \phi+a(c / D)^{1 / 2}\right]$, assuming a constant value for cohesion. We find experimentally that cohesion increases with depth, and we have accommodated this fact to the theory. Using this form of the theory to analyze the results for the avalanche angle, we find a good fit to the experimental data for narrow beds. We have developed further extensions to the method of wedges, including twoand three-segment slip planes, partially pinned to the retaining walls. The fit between theoretical results and the experimental results of the angle of avalanche and avalanche depth for Canon CLC500 is quite good in the whole range of bed widths for a value of the angle of internal friction of $10^{\circ}$. We have also analyzed a series of model xerographic toners differing in the amount of surface additives and the values of the angle of internal friction range from $8^{\circ}$ to $23^{\circ}$. We have found that the angle of internal friction increases with toner bulk density. These angles are smaller than the typical values of smooth noncohesive grains. Although there is no clear explanation for this result, the low density of fine cohesive powders, due to the high values of their free volume, as compared with the typical densities of smooth noncohesive 
grains may be the reason for such low values of the angle of internal friction.

\section{ACKNOWLEDGMENTS}

We are indebted to Frank Genovesse for suggesting the powder replication technique used for measuring that post- avalanche profiles of the xerographic toners in this study. We thank Mike Morgan for his help with the experimental measurements. We also thank Enrique Viturro for fruitful discussions. This research was supported by the Xerox Foundation, Xerox Corporation and by the Spanish Government Agency, Dirección General de Ciencia y Tecnología (DGES) under Contract No. PB96-1375.
[1] H. M. Jaeger, C. H. Liu, and S. R. Nagel, Phys. Rev. Lett. 62, 40 (1988)

[2] K. M. Hill, A. Caprihan, and J. Kakalios, Phys. Rev. Lett. 78, 50 (1997).

[3] T. C. Halsey and A. J. Levine, Phys. Rev. Lett. 80, 3141 (1998).

[4] H. M. Jaeger and S. R. Nagel, Science 255, 1523 (1992).

[5] D. J. Hornbaker, R. Albert, I. Albert, A.-L. Barabasi, and P. Schiffer, Nature (London) 387, 765 (1997).

[6] R. Albert, I. Albert, D. Hornbaker, P. Schiffer, and A. L. Barabasi, Phys. Rev. E 56, R6271 (1997).

[7] L. Bocquet, E. Charlaix, S. Ciliberto, and J. Crassons, Nature (London) 396, 735 (1998).

[8] H. Schubert, Powder Technol. 37, 105 (1984).

[9] H. C. Hamaker, Physica (Amsterdam) 4, 1058 (1937).

[10] J. N. Israelachvili and D. Tabor, Proc. R. Soc. London, Ser. A 31, 19 (1972).

[11] H. Krupp, Adv. Coll. Interface Sci. 1, 111 (1967).

[12] J. M. Valverde, A. Ramos, A. Castellanos, and P. K. Watson, Powder Technol. 97, 237 (1998).

[13] J. M. Valverde, A. Castellanos, and P. K. Watson, Powder Technol. (to be published).

[14] A. Castellanos, J. M. Valverde, and P. K. Watson, Z. Angew. Math. Mech. 80, 423 (2000).
[15] C. A. Coulomb, Mémoires de Mathématics et de Physique Présentés à l'Académie des Sciences par Divers Savants et lus Dans les Sssemblées, Année 1773 (Académie Royale des Sciences, Paris, 1776), Vol. 7, p. 343.

[16] K. Rietema, The Dynamics of Fine Powders (Elsevier, London, 1991).

[17] P. K. Watson, A. Castellanos, A. Ramos, and J. M. Valverde, Proceedings of the 19th Annual Meeting of the Adhesion Society, February 18-21, edited by T. C. Ward (Adhesion Society, Myrtle Beach, South Carolina, 1996), pp. 338-398.

[18] R. Nedderman, Statics and Kinematics of Granular Materials (Cambridge University Press, Cambridge, 1992).

[19] B. J. Briscoe and M. J. Adams, Tribology in Particle Technology (Hilger, Bristol, 1987).

[20] O. Molerus, Powder Technol. 33, 81 (1982).

[21] Disorder and Granular Media, edited by D. Bideau and A. Hansen (Elsevier, Amsterdam, 1993).

[22] A. Castellanos, J. M. Valverde, A. T. Pérez, A. Ramos, and P. K. Watson, Phys. Rev. Lett. 82, 1156 (1999).

[23] K. M. Schmidt and D. R. Montgomery, Science 270, 617 (1995).

[24] J. H. Atkinson, Introduction to the Mechanics of Soils and Foundations (McGraw-Hill, New York, 1993). 